\title{
Reaction monitoring by Benchtop NMR spectroscopy using a novel stationary flow reactor setup
}

\section{-Supporting Information-}

\author{
Anne Friebel, Erik von Harbou, \\ Kerstin Münnemann, Hans Hasse
}

Laboratory of Engineering Thermodynamics, University of Kaiserslautern, Erwin-Schrödinger-Straße 44, 67663 Kaiserslautern, Germany 


\section{S1 Kinetic model of Dörrhöfer}

The kinetic model for the formation of methyl acetate is defined by Dörrhöfer et al. [1] as follows:

$$
r=\frac{x_{\mathrm{H} 2 \mathrm{SO} 4}^{\alpha}}{x_{\mathrm{W}}^{0.5}} \cdot\left[k_{\mathrm{f}} \cdot a_{\mathrm{MeOH}} \cdot a_{\mathrm{AA}}-k_{\mathrm{b}} \cdot a_{\mathrm{MA}} \cdot a_{\mathrm{W}}\right]
$$

$\alpha$ is the catalyst parameter which is adjustable. The activity coefficients $\gamma_{\mathrm{i}}$ were calculated using the UNIQUAC model with parameters from Pöpken et al. [2]. The kinetic constants are defined as follows:

$$
\begin{aligned}
& k_{\mathrm{f}}=k_{0 \mathrm{f}} \cdot \exp \left(\frac{-E_{\mathrm{Af}}}{R T}\right) \\
& k_{\mathrm{b}}=k_{0 \mathrm{~b}} \cdot \exp \left(\frac{-E_{\mathrm{Ab}}}{R T}\right)
\end{aligned}
$$

$k_{0 \mathrm{f}}, k_{0 \mathrm{~b}}, E_{\mathrm{Af}}$, and $E_{\mathrm{Ab}}$ are the four adjustable kinetic parameters. By fitting the model to the experimental data using the Runge-Kutta method the parameters were obtained which are listed in Table S1

\section{S2 Premagnetization in flow}

To estimate the maximum volume flow rate in the setup, the component specific signal intensity in the ${ }^{1} \mathrm{H}$ NMR spectrum as a function of the volume flow rate was investigated. Therefore the stationary flow setup was used with one feed line. For each component the following steps were performed: the setup was filled with the pure component, a ${ }^{1} \mathrm{H}$ NMR spectrum at $\dot{V}=0 \mathrm{ml} / \mathrm{min}$ was measured (equilibrium magnetization which gives the maximum signal intensity), the flow rate was increased to the next value and was applied for at least 5 min to ensure steady-state, the corresponding ${ }^{1} \mathrm{H}$ NMR spectrum was measured. For each flow rate, three independent ${ }^{1} \mathrm{H}$ NMR measurements were performed at intervals of $60 \mathrm{~s}$.

\section{S3 RTD measurements}

\section{S3.1 Procedure}

The residence time distribution was measured in a step response setup which is shown in Figure S1. It consists of two high-pressure dosage pumps delivering the feeds A (water) and $\mathrm{B}$ (water with tracer) and two Coriolis flow sensors which are regulating the mass flow of the pumps. Both stream lines were connected to a six-port-two-position-valve. Position 1: Feed A flows through the NMR, Feed B flows to a waste bottle and position 2: the other way around. Hence, a concentration step was introduced in the flow setup by switching the valve from position 1 to position 2 . All feed lines were capillaries made of PEEK $\left(d_{\mathrm{i}}=1 \mathrm{~mm}\right)$. 
As tracer, acetonitrile (Carl Roth, Rotisolv, purity: $\geq 0.999 \mathrm{~g} / \mathrm{g}$ ) or methanol (Carl Roth, Anhydrous, purity: $\geq 0.998 \mathrm{~g} / \mathrm{g}$ ) with a mole fraction of $x_{\text {Tracer }}=0.07 \mathrm{~mol} / \mathrm{mol}$ in water was used. All chemicals were degassed before the experiment. To obtain a steady-state inside the feed lines the pumps were operated for $5 \mathrm{~min}$. Then the valve was switched automatically. This time-stamp marks the beginning of the experiment and corresponds to $t_{0}^{\operatorname{Exp}}$. After a time interval that was chosen according to the reactor length and flow rate, the repeated ${ }^{1} \mathrm{H}$ NMR measurement was started. This time-stamp marks the beginning of the analysis and corresponds to $t_{0}^{\mathrm{NMR}}$. After the step response has been recorded with the NMR spectrometer the valve was switched again to flush the system with water for $5 \mathrm{~min}$. Each measurement was repeated three times. The mean residence time $\bar{t}$ is defined as the point where $50 \%$ of the integral under the F-curve is reached and was determined from the experimental data.

Residence time distribution measurements were performed with a fast repeating ${ }^{1} \mathrm{H}$ measurement with intervals of $6 \mathrm{~s}$ between the spectra (acquisition time: $3.2 \mathrm{~s}, 16 \mathrm{k}$ data points, $90^{\circ}$ pulse, one scan).

\section{S3.2 Parametrization of TSR model}

In Figure $\mathrm{S} 2$ the reactor position $L_{\mathrm{R}}$ is plotted as a function of mean residence time $\bar{t}$ (determined from the RTD measurements) for the two applied flow rates and acetonitrile as concentration step. A straight line was fitted to the measured data points and they are in very good agreement to it. Thus, the mean residence times for the other reactor lengths were calculated with the linear fit. The slope of the linear fit is in accordance to the considered mean velocity in the reactor. In Table S3 the investigated reactor positions and the corresponding mean residence times are listed. The reactor positions indicated with * are those investigated with RTD measurements.

The number of tanks resulting from the TSR model fit are plotted in Figure S3 as a function of mean residence time $\bar{t}$ in the reactor for both flow rates. Reactor length $\mathrm{R} 4$, R7 and R9 show a linear dependence, only reactor length R2 underruns the expected number of tanks. This deviation is most likely attributable to inflow effects. The fitted straight lines are forced through the zero point, $\mathrm{R} 2$ is not taken into account for both fits. The slope of the linear fit is dependent on the self-diffusion coefficient [3, 4] of acetonitrile in water and results in a value of 2.8 and $2.410^{-9} \mathrm{~m}^{2} / \mathrm{s}$, respectively. The differences can be explained by the tracer concentration not being infinitely diluted. Table S3 shows the investigated reactor positions and the corresponding number of tanks. The reactor positions indicated with $*$ are those measured with residence distribution measurements. The number of tanks at the other reactor positions were calculated with the linear fit.

\section{S3.3 Sensitivity study 'PFR'}

It was investigated how the chosen reactor model influences the fitted rate constant $k_{\mathrm{f}}$ in the reactor capillary. In comparison to the TSR model the PFR model neglects the influence of back-mixing and dispersion on the conversion of reactants. For the PFR the derivative of the extent of reaction $\lambda^{\text {mod,PFR }}$ with respect to the position $z$ in the reactor 
is linked to the rate of reaction $r$ as follows in Equation S3:

$$
\frac{d \lambda^{\text {mod,PFR }}}{d z}=\frac{A_{i}}{\dot{V}} \cdot r
$$

wherein $\dot{V}$ is the invariant volume flow rate and $A_{i}$ the inner cross-sectional area of the reactor.

For the sensitivity study an hypothetical data set with known rate constants was fitted with either TSR or PFR as reactor model. The rate constants resulting from both fits were compared. The solution of the TSR fit was set to be the basic truth. In Figure S4 the relative error of rate constant $k_{\mathrm{f}}$ (using PFR instead of TSR) is plotted as a function of $k_{\mathrm{f}}$ and of the time constant $t_{1 / 2}$ (time when half of the conversion that would be obtained in equilibrium is reached) for the two investigated flow rates. The faster the reaction, the larger the relative error of rate constant $k_{\mathrm{f}}$. For the reactions investigated in this work the relative error of rate constant $k_{\mathrm{f}}$ is small $(<8 \%)$ when applying the PFR model instead of the TSR model for the kinetic fit. Hence, the use of the PFR model would be reasonable for the fitting of kinetic measurements in this work as well.

\section{S4 Experimental data}

\section{S4.1 Kinetic data}

In Table S4 and Table S5 the experimental data of the static tube experiments of both esterification reactions are listed. The stationary flow experimental data is listed in Tables S6 and Table S7. Table S8 shows the averaged mean values that were used for the kinetic evaluation.

\section{References}

[1] T. Dörrhöfer, Gestaltung und Effektivität von Bodenkolonnen für die Reaktivrektifikation, Ph.D. thesis, TU München, 2006.

[2] T. Pöpken, L. Götze, J. Gmehling, Reaction Kinetics and Chemical Equilibrium of Homogeneously and Heterogeneously Catalyzed Acetic Acid Esterification with Methanol and Methyl Acetate Hydrolysis, Ind. Eng. Chem. Res. 39 (7) (2000) 26012611. doi:10.1021/ie000063q.

[3] G. Taylor, Dispersion of Soluble Matter in Solvent Flowing Slowly through a Tube, Proceedings of the Royal Society of London. Series A, Mathematical and Physical Sciences 219 (1137) (1953) 186-203.

[4] R. Aris, On the Dispersion of a Solute in a Fluid Flowing through a Tube, Proceedings of the Royal Society of London. Series A, Mathematical and Physical Sciences 235 (1200) (1956) 67-77. 


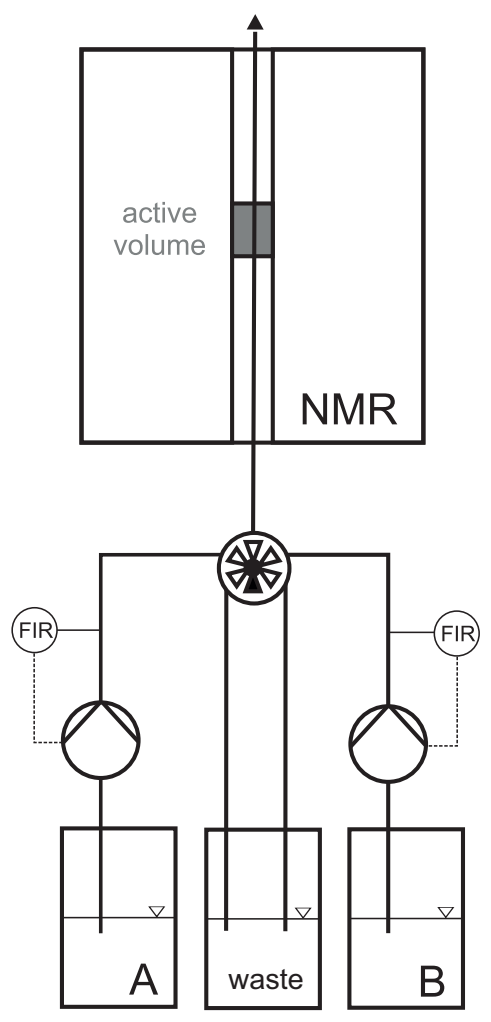

Figure S 1: Experimental setup of the residence time distribution measurement.

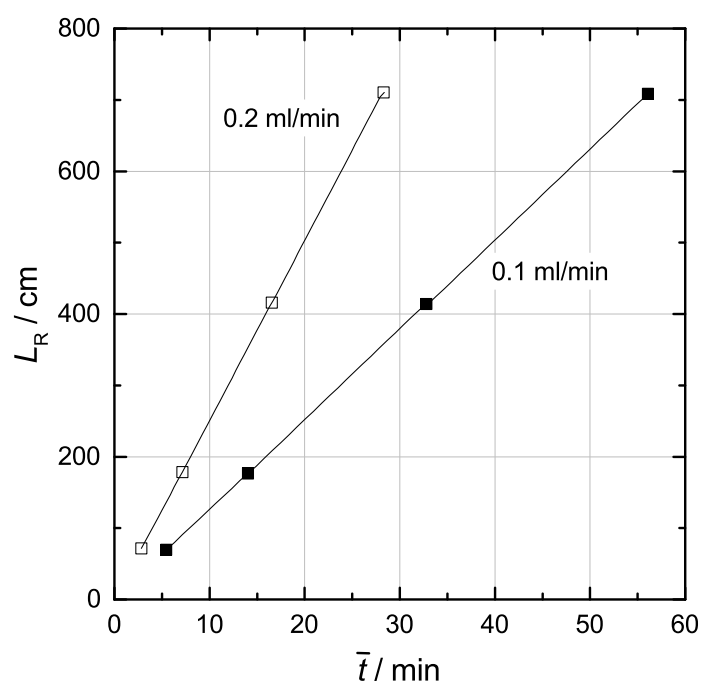

Figure S 2: Reactor position as a function of mean residence time for two different flow rates. Symbols: Experimental data of present work; Lines: Linear fit. 


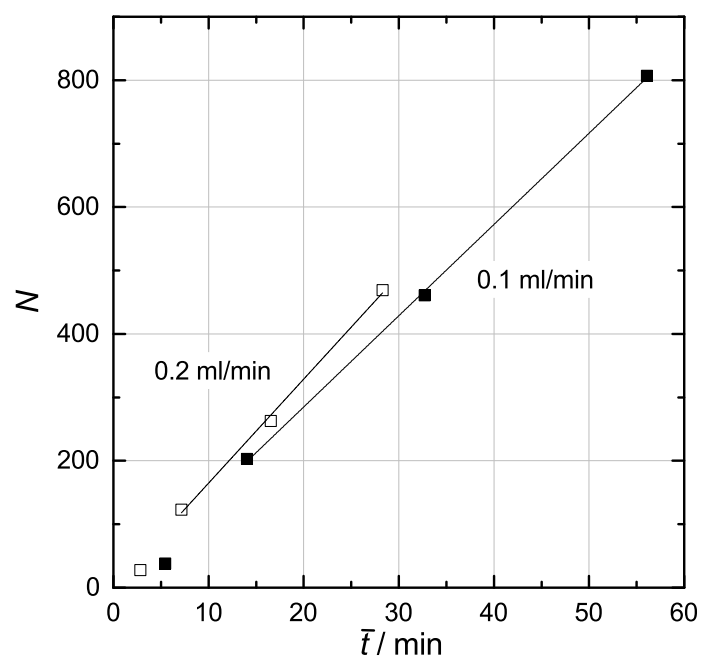

Figure S 3: Number of tanks (TSR model) as a function of mean residence time for two different flow rates. Symbols: Experimental data of present work; Lines: Linear fit.

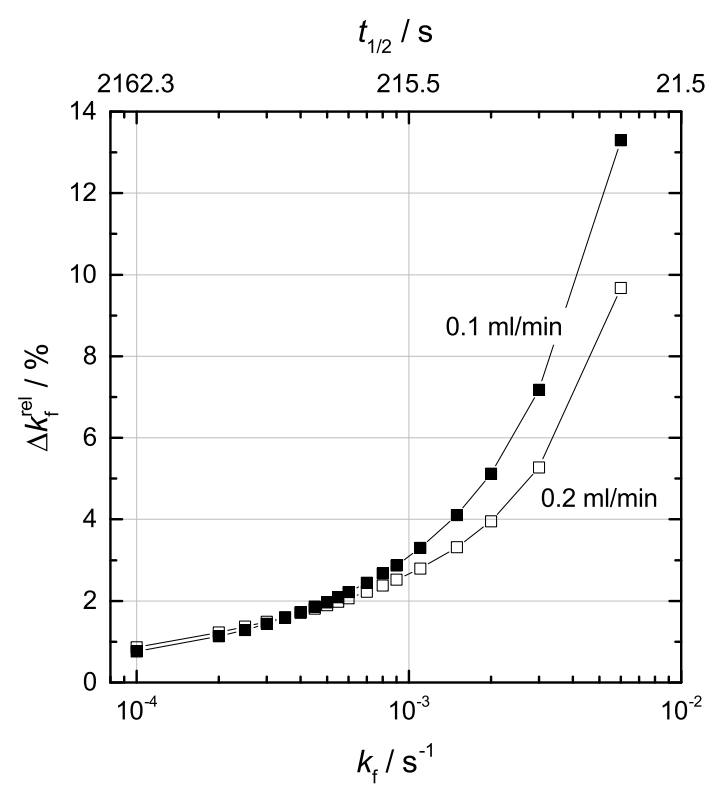

Figure S 4: Relative error of rate constant $k_{\mathrm{f}}$ when assuming PFR instead of TSR as a function of $k_{\mathrm{f}}$ and time constant $t_{1 / 2}$ for both flow rates. 
Table S 1: Adjustable parameters of the reaction kinetic model of Dörrhöfer [1].

\begin{tabular}{ccccc}
\hline $\begin{array}{c}k_{0 \mathrm{f}} \\
\mathrm{s}^{-1}\end{array}$ & $\begin{array}{c}k_{0 \mathrm{~b}} \\
\mathrm{~s}^{-1}\end{array}$ & $\begin{array}{c}E_{\mathrm{Af}} \\
\mathrm{kJ} \mathrm{mol}\end{array}$ & $\begin{array}{c}E_{\mathrm{Ab}} \\
\mathrm{kJ} \mathrm{mol}^{-1}\end{array}$ & $\alpha$ \\
\hline $3.01 \cdot 10^{5}$ & $7.58 \cdot 10^{4}$ & 41.69 & 47.53 & 0.781 \\
\hline
\end{tabular}

Table S 2: Default parameter for the used pulse sequences.

\begin{tabular}{ll}
\hline T1 inversion recovery & \\
Bandwidth & $5 \mathrm{kHz}$ \\
90 pulse width & $7 \mu \mathrm{s}$ \\
Dwell time & $200 \mu \mathrm{s}$ \\
Number of points & $32 \mathrm{k}$ \\
\hline 1H periodic & \\
Bandwidth & $5 \mathrm{kHz}$ \\
90 pulse width & $7 \mu \mathrm{s}$ \\
Dwell time & $200 \mu \mathrm{s}$ \\
\hline COSY & \\
Number of points t1 / t2 & $256 / 8,192$ \\
t1 increment & $1.43 \mathrm{~ms}$ \\
Bandwidth f1 / f2 & $0.7 / 5 \mathrm{kHz}$ \\
Dwell time & $200 \mu \mathrm{s}$ \\
Acq. / Rep. time & $1.638 / 2 \mathrm{~s}$ \\
\hline HETCOR & \\
Number of points t1 / t2 & $64 / 4,096$ \\
t1 increment & $1 \mathrm{~ms}$ \\
Bandwidth f1 / f2 & $1 / 2.5 \mathrm{kHz}$ \\
Dwell time & $400 \mu \mathrm{s}$ \\
Single bond delay & $3.6 \mathrm{~ms}(140 \mathrm{~Hz})$ \\
Multiple bond delay & $2.4 \mathrm{~ms}(10 \mathrm{~Hz})$ \\
\hline HMBC & \\
Number of points t1 / t2 & $128 / 1,024$ \\
t1 increment & $0.4 \mathrm{~ms}$ \\
Bandwidth f1 / f2 & $2.5 / 2 \mathrm{kHz}$ \\
Dwell time & $500 \mu \mathrm{s}$ \\
Single bond delay & $3.6 \mathrm{~ms}(140 \mathrm{~Hz})$ \\
Multiple bond delay & $50 \mathrm{~ms}(10 \mathrm{~Hz})$ \\
\hline &
\end{tabular}


Table S 3: Reactor position, mean residence times and corresponding number of tanks for different flow rates. ${ }^{*}$ measured, other ones calculated with linear fit.

\begin{tabular}{lcccccccc}
\hline & \multicolumn{9}{c}{$0.1 \mathrm{ml} / \mathrm{min}$} & \multicolumn{6}{c}{$0.2 \mathrm{ml} / \mathrm{min}$} \\
$z_{i}$ & $L_{R} / \mathrm{cm}$ & $\bar{t} / \min$ & $N^{\exp }$ & $N^{\text {calc }}$ & $\bar{t} / \min$ & $N^{\exp }$ & $N^{\text {calc }}$ & tracer \\
\hline$z_{1}$ & 26.0 & 1.98 & & 28 & 1.07 & & 18 & $\mathrm{ACN}$ \\
$z_{2}{ }^{*}$ & 70.7 & 5.57 & 38 & 80 & 2.89 & 28 & 47 & $\mathrm{ACN}$ \\
$z_{3}$ & 120.7 & 9.49 & & 136 & 4.85 & & 80 & $\mathrm{ACN}$ \\
$z_{4}{ }^{*}$ & 177.4 & 14.05 & 203 & 201 & 7.14 & 123 & 117 & $\mathrm{ACN}$ \\
$z_{5}$ & 242.9 & 19.16 & & 274 & 9.72 & & 160 & $\mathrm{ACN}$ \\
$z_{6}$ & 320.3 & 25.29 & & 362 & 12.80 & & 210 & $\mathrm{ACN}$ \\
$z_{7}{ }^{*}$ & 415.0 & 32.72 & 461 & 468 & 16.55 & 263 & 272 & $\mathrm{ACN}$ \\
$z_{8}$ & 537.2 & 42.47 & & 608 & 21.44 & & 352 & $\mathrm{ACN}$ \\
$z_{9}{ }^{*}$ & 709.3 & 56.09 & 807 & 803 & 28.30 & 469 & 465 & $\mathrm{ACN}$ \\
$z_{10}$ & 1003.6 & 79.42 & & 1137 & 40.02 & & 658 & $\mathrm{ACN}$ \\
\hline$z_{2}{ }^{*}$ & 70.7 & 5.51 & - & & 2.84 & - & & $\mathrm{MeOH}$ \\
\hline
\end{tabular}


Table S 4: Experimental data of static tube experiments with methanol and formic acid.

\begin{tabular}{cccccc}
\hline \multicolumn{2}{c}{$0 \mathrm{ma} \%$} & \multicolumn{2}{c}{$2 \mathrm{ma} \%$} & \multicolumn{2}{c}{$2 \mathrm{ma} \%$} \\
$\mathrm{t} / \mathrm{min}$ & $\lambda$ & $\mathrm{t} / \mathrm{min}$ & $\lambda$ & $\mathrm{t} / \mathrm{min}$ & $\lambda$ \\
\hline 1.50 & 0.0327 & 1.36 & 0.2399 & 42.36 & 0.3608 \\
3.50 & 0.0715 & 2.36 & 0.2822 & 43.36 & 0.3606 \\
5.50 & 0.0969 & 3.36 & 0.3025 & 44.36 & 0.3603 \\
7.50 & 0.1171 & 4.36 & 0.3157 & 45.36 & 0.3612 \\
9.50 & 0.1327 & 5.36 & 0.3248 & 46.36 & 0.3614 \\
11.50 & 0.1450 & 6.36 & 0.3310 & 47.36 & 0.3604 \\
13.50 & 0.1564 & 7.36 & 0.3369 & 48.36 & 0.3610 \\
15.50 & 0.1651 & 8.36 & 0.3407 & 49.36 & 0.3608 \\
17.50 & 0.1725 & 9.36 & 0.3444 & 50.36 & 0.3606 \\
19.50 & 0.1797 & 10.36 & 0.3461 & 51.36 & 0.3595 \\
21.50 & 0.1858 & 11.36 & 0.3492 & 52.36 & 0.3609 \\
23.50 & 0.1912 & 12.36 & 0.3514 & 53.36 & 0.3604 \\
25.50 & 0.1958 & 13.36 & 0.3527 & 54.36 & 0.3611 \\
27.50 & 0.2010 & 14.36 & 0.3542 & 55.36 & 0.3604 \\
29.50 & 0.2044 & 15.36 & 0.3545 & 56.36 & 0.3610 \\
31.50 & 0.2090 & 16.36 & 0.3558 & 57.36 & 0.3607 \\
33.50 & 0.2123 & 17.36 & 0.3564 & 58.36 & 0.3612 \\
35.50 & 0.2155 & 18.36 & 0.3569 & 59.36 & 0.3606 \\
37.50 & 0.2197 & 19.36 & 0.3573 & 60.36 & 0.3604 \\
39.50 & 0.2223 & 20.36 & 0.3584 & 61.36 & 0.3607 \\
41.50 & 0.2258 & 21.36 & 0.3586 & 62.36 & 0.3609 \\
43.50 & 0.2277 & 22.36 & 0.3592 & 63.36 & 0.3610 \\
45.50 & 0.2301 & 23.36 & 0.3588 & 64.36 & 0.3606 \\
47.50 & 0.2324 & 24.36 & 0.3599 & 65.36 & 0.3604 \\
49.50 & 0.2358 & 25.36 & 0.3596 & 66.36 & 0.3605 \\
51.50 & 0.2379 & 26.36 & 0.3596 & 67.36 & 0.3607 \\
53.50 & 0.2392 & 27.36 & 0.3598 & 68.36 & 0.3601 \\
55.50 & 0.2415 & 28.36 & 0.3601 & 69.36 & 0.3615 \\
57.50 & 0.2427 & 29.36 & 0.3601 & 70.36 & 0.3612 \\
59.50 & 0.2460 & 30.36 & 0.3604 & 71.36 & 0.3605 \\
61.50 & 0.2470 & 31.36 & 0.3609 & 72.36 & 0.3600 \\
63.50 & 0.2483 & 32.36 & 0.3618 & 73.36 & 0.3613 \\
65.50 & 0.2497 & 33.36 & 0.3599 & 74.36 & 0.3610 \\
67.50 & 0.2517 & 34.36 & 0.3602 & 75.36 & 0.3611 \\
69.50 & 0.2534 & 35.36 & 0.3605 & 76.36 & 0.3609 \\
71.50 & 0.2543 & 36.36 & 0.3611 & 77.36 & 0.3609 \\
73.50 & 0.2553 & 37.36 & 0.3615 & 78.36 & 0.3601 \\
75.50 & 0.2575 & 38.36 & 0.3603 & 79.36 & 0.3613 \\
77.50 & 0.2581 & 39.36 & 0.3609 & 80.36 & 0.3606 \\
79.50 & 0.2599 & 40.36 & 0.3603 & & \\
81.50 & 0.2604 & 41.36 & 0.3611 & & \\
\hline & & & & &
\end{tabular}


Table S 5: Experimental data of static tube experiments with methanol and acetic acid.

\begin{tabular}{|c|c|c|c|c|c|c|c|}
\hline \multicolumn{2}{|c|}{$1.3 \mathrm{ma} \%$} & \multicolumn{2}{|c|}{$1.3 \mathrm{ma} \%$} & \multicolumn{2}{|c|}{$3.5 \mathrm{ma} \%$} & \multicolumn{2}{|c|}{$3.5 \mathrm{ma} \%$} \\
\hline $\mathrm{t} / \min$ & $\lambda$ & $\mathrm{t} / \min$ & $\lambda$ & $\mathrm{t} / \min$ & $\lambda$ & $\mathrm{t} / \min$ & $\lambda$ \\
\hline 1.27 & 0.0318 & 42.27 & 0.1981 & 1.03 & 0.05495 & 42.03 & 0.28856 \\
\hline 2.27 & 0.0434 & 43.27 & 0.2000 & 2.03 & 0.07926 & 43.03 & 0.29011 \\
\hline 3.27 & 0.0528 & 44.27 & 0.2019 & 3.03 & 0.10035 & 44.03 & 0.29009 \\
\hline 4.27 & 0.0612 & 45.27 & 0.2039 & 4.03 & 0.11887 & 45.03 & 0.29185 \\
\hline 5.27 & 0.0696 & 46.27 & 0.2048 & 5.03 & 0.13362 & 46.03 & 0.29351 \\
\hline 6.27 & 0.0770 & 47.27 & 0.2066 & 6.03 & 0.14558 & 47.03 & 0.2949 \\
\hline 7.27 & 0.0837 & 48.27 & 0.2085 & 7.03 & 0.1574 & 48.03 & 0.29588 \\
\hline 8.27 & 0.0895 & 49.27 & 0.2102 & 8.03 & 0.1683 & 49.03 & 0.29762 \\
\hline 9.27 & 0.0967 & 50.27 & 0.2111 & 9.03 & 0.17671 & 50.03 & 0.29881 \\
\hline 10.27 & 0.1021 & 51.27 & 0.2125 & 10.03 & 0.18374 & 51.03 & 0.29903 \\
\hline 11.27 & 0.1072 & 52.27 & 0.2135 & 11.03 & 0.19283 & 52.03 & 0.29922 \\
\hline 12.27 & 0.1111 & 53.27 & 0.2151 & 12.03 & 0.1997 & 53.03 & 0.30111 \\
\hline 13.27 & 0.1169 & 54.27 & 0.2171 & 13.03 & 0.20546 & 54.03 & 0.30194 \\
\hline 14.27 & 0.1220 & 55.27 & 0.2175 & 14.03 & 0.21155 & 55.03 & 0.30299 \\
\hline 15.27 & 0.1262 & 56.27 & 0.2189 & 15.03 & 0.21746 & 56.03 & 0.30344 \\
\hline 16.27 & 0.1306 & 57.27 & 0.2204 & 16.03 & 0.22166 & 57.03 & 0.30432 \\
\hline 17.27 & 0.1342 & 58.27 & 0.2215 & 17.03 & 0.2257 & 58.03 & 0.30488 \\
\hline 18.27 & 0.1373 & 59.27 & 0.2230 & 18.03 & 0.23077 & 59.03 & 0.30739 \\
\hline 19.27 & 0.1412 & 60.27 & 0.2243 & 19.03 & 0.23465 & 60.03 & 0.3072 \\
\hline 20.27 & 0.1448 & 61.27 & 0.2248 & 20.03 & 0.23905 & 61.03 & 0.30819 \\
\hline 21.27 & 0.1477 & 62.27 & 0.2264 & 21.03 & 0.24138 & 62.03 & 0.30953 \\
\hline 22.27 & 0.1513 & 63.27 & 0.2266 & 22.03 & 0.24525 & 63.03 & 0.30986 \\
\hline 23.27 & 0.1548 & 64.27 & 0.2281 & 23.03 & 0.24829 & 64.03 & 0.31077 \\
\hline 24.27 & 0.1579 & 65.27 & 0.2299 & 24.03 & 0.25104 & 65.03 & 0.31104 \\
\hline 25.27 & 0.1611 & 66.27 & 0.2309 & 25.03 & 0.25455 & 66.03 & 0.31153 \\
\hline 26.27 & 0.1636 & 67.27 & 0.2318 & 26.03 & 0.25885 & 67.03 & 0.31262 \\
\hline 27.27 & 0.1670 & 68.27 & 0.2326 & 27.03 & 0.25997 & 68.03 & 0.31375 \\
\hline 28.27 & 0.1691 & 69.27 & 0.2333 & 28.03 & 0.26219 & 69.03 & 0.31397 \\
\hline 29.27 & 0.1715 & 70.27 & 0.2354 & 29.03 & 0.26495 & 70.03 & 0.31418 \\
\hline 30.27 & 0.1745 & 71.27 & 0.2358 & 30.03 & 0.26766 & 71.03 & 0.31497 \\
\hline 31.27 & 0.1768 & 72.27 & 0.2361 & 31.03 & 0.26907 & 72.03 & 0.31631 \\
\hline 32.27 & 0.1787 & 73.27 & 0.2376 & 32.03 & 0.27124 & 73.03 & 0.31591 \\
\hline 33.27 & 0.1811 & 74.27 & 0.2386 & 33.03 & 0.27317 & 74.03 & 0.31689 \\
\hline 34.27 & 0.1833 & 75.27 & 0.2391 & 34.03 & 0.27542 & 75.03 & 0.31759 \\
\hline 35.27 & 0.1860 & 76.27 & 0.2404 & 35.03 & 0.27791 & 76.03 & 0.31902 \\
\hline 36.27 & 0.1875 & 77.27 & 0.2412 & 36.03 & 0.27963 & 77.03 & 0.31843 \\
\hline 37.27 & 0.1897 & 78.27 & 0.2419 & 37.03 & 0.28073 & 78.03 & 0.3196 \\
\hline 38.27 & 0.1911 & 79.27 & 0.2431 & 38.03 & 0.28247 & 79.03 & 0.31979 \\
\hline 39.27 & 0.1931 & 80.27 & 0.2437 & 39.03 & 0.28389 & 80.03 & 0.31962 \\
\hline 40.27 & 0.1951 & & & 40.03 & 0.28469 & & \\
\hline 41.27 & 0.1973 & & & 41.03 & 0.2865 & & \\
\hline
\end{tabular}


Table S 6: Experimental data of stationary flow experiments with methanol and formic acid.

\begin{tabular}{|c|c|c|c|c|c|c|c|c|c|}
\hline \multicolumn{2}{|c|}{$0 \mathrm{ma} \%$} & \multicolumn{2}{|c|}{0 ma\% } & \multicolumn{2}{|c|}{$0 \mathrm{ma} \%$} & \multicolumn{2}{|c|}{0 ma\% } & \multicolumn{2}{|c|}{0 ma\% } \\
\hline $\mathrm{t} / \min$ & $\lambda$ & $\mathrm{t} / \min$ & $\lambda$ & $\mathrm{t} / \min$ & $\lambda$ & $\mathrm{t} / \min$ & $\lambda$ & $\mathrm{t} / \min$ & $\lambda$ \\
\hline 1.98 & 0.0439 & 9.49 & 0.1347 & 19.16 & 0.1810 & 32.72 & 0.2121 & 56.09 & 0.2386 \\
\hline 1.98 & 0.0465 & 9.49 & 0.1391 & 19.16 & 0.1802 & 32.72 & 0.2124 & 56.09 & 0.2394 \\
\hline 1.98 & 0.0466 & 9.49 & 0.1357 & 19.16 & 0.1809 & 32.72 & 0.2122 & 56.09 & 0.2392 \\
\hline 1.98 & 0.0431 & 9.49 & 0.1317 & 19.16 & 0.1781 & 32.72 & 0.2129 & 56.09 & 0.2389 \\
\hline 1.98 & 0.0426 & 9.49 & 0.1296 & 19.16 & 0.1819 & 32.72 & 0.2119 & 56.09 & 0.2392 \\
\hline 1.98 & 0.0425 & 9.49 & 0.1370 & 19.16 & 0.1791 & 32.72 & 0.2107 & 56.09 & 0.2388 \\
\hline 1.98 & 0.0417 & 9.49 & 0.1375 & 19.16 & 0.1777 & 32.72 & 0.2115 & 56.09 & 0.2401 \\
\hline 1.98 & 0.0428 & 9.49 & 0.1349 & 19.16 & 0.1809 & 32.72 & 0.2122 & 56.09 & 0.2404 \\
\hline 1.98 & 0.0436 & 9.49 & 0.1377 & 19.16 & 0.1808 & 32.72 & 0.2137 & 56.09 & 0.2395 \\
\hline 1.98 & 0.0429 & 9.49 & 0.1370 & 19.16 & 0.1798 & 32.72 & 0.2132 & 56.09 & 0.2395 \\
\hline 5.57 & 0.0994 & 14.05 & 0.1596 & 25.29 & 0.1996 & 42.47 & 0.2303 & 79.42 & 0.2582 \\
\hline 5.57 & 0.0974 & 14.05 & 0.1615 & 25.29 & 0.1961 & 42.47 & 0.2309 & 79.42 & 0.2585 \\
\hline 5.57 & 0.0978 & 14.05 & 0.1577 & 25.29 & 0.1914 & 42.47 & 0.2307 & 79.42 & 0.2575 \\
\hline 5.57 & 0.0971 & 14.05 & 0.1642 & 25.29 & 0.1975 & 42.47 & 0.2296 & 79.42 & 0.2577 \\
\hline 5.57 & 0.0964 & 14.05 & 0.1583 & 25.29 & 0.1958 & 42.47 & 0.2284 & 79.42 & 0.2590 \\
\hline 5.57 & 0.0989 & 14.05 & 0.1588 & 25.29 & 0.1955 & 42.47 & 0.2294 & 79.42 & 0.2588 \\
\hline 5.57 & 0.0996 & 14.05 & 0.1572 & 25.29 & 0.1955 & 42.47 & 0.2283 & 79.42 & 0.2584 \\
\hline 5.57 & 0.0987 & 14.05 & 0.1602 & 25.29 & 0.1947 & 42.47 & 0.2272 & 79.42 & 0.2583 \\
\hline 5.57 & 0.0983 & 14.05 & 0.1616 & 25.29 & 0.1952 & 42.47 & 0.2276 & 79.42 & 0.2582 \\
\hline 5.57 & 0.0977 & 14.05 & 0.1625 & 25.29 & 0.1923 & 42.47 & 0.2271 & 79.42 & 0.2576 \\
\hline \multicolumn{2}{|c|}{2 ма\% } & \multicolumn{2}{|c|}{2 ma\% } & \multicolumn{2}{|c|}{$2 \mathrm{ma} \%$} & \multicolumn{2}{|c|}{$2 \mathrm{ma} \%$} & \multicolumn{2}{|c|}{$2 \mathrm{ma} \%$} \\
\hline $\mathrm{t} / \min$ & $\lambda$ & $\mathrm{t} / \min$ & $\lambda$ & $\mathrm{t} / \min$ & $\lambda$ & $\mathrm{t} / \min$ & $\lambda$ & $\mathrm{t} / \min$ & $\lambda$ \\
\hline 1.98 & 0.2641 & 9.49 & 0.3401 & 19.16 & 0.3527 & 32.72 & 0.3580 & 56.09 & 0.3598 \\
\hline 1.98 & 0.2634 & 9.49 & 0.3415 & 19.16 & 0.3539 & 32.72 & 0.3566 & 56.09 & 0.3589 \\
\hline 1.98 & 0.2625 & 9.49 & 0.3380 & 19.16 & 0.3554 & 32.72 & 0.3557 & 56.09 & 0.3580 \\
\hline 1.98 & 0.2650 & 9.49 & 0.3374 & 19.16 & 0.3513 & 32.72 & 0.3561 & 56.09 & 0.3593 \\
\hline 1.98 & 0.2640 & 9.49 & 0.3425 & 19.16 & 0.3505 & 32.72 & 0.3553 & 56.09 & 0.3582 \\
\hline 1.98 & 0.2634 & 9.49 & 0.3385 & 19.16 & 0.3496 & 32.72 & 0.3585 & 56.09 & 0.3581 \\
\hline 1.98 & 0.2626 & 9.49 & 0.3381 & 19.16 & 0.3522 & 32.72 & 0.3590 & 56.09 & 0.3571 \\
\hline 1.98 & 0.2621 & 9.49 & 0.3377 & 19.16 & 0.3551 & 32.72 & 0.3553 & 56.09 & 0.3565 \\
\hline 1.98 & 0.2619 & 9.49 & 0.3385 & 19.16 & 0.3533 & 32.72 & 0.3580 & 56.09 & 0.3553 \\
\hline 1.98 & 0.2639 & 9.49 & 0.3393 & 19.16 & 0.3559 & 32.72 & 0.3544 & 56.09 & 0.3565 \\
\hline 5.57 & 0.3256 & 14.05 & 0.3519 & 25.29 & 0.3514 & 42.47 & 0.3558 & 79.42 & 0.3567 \\
\hline 5.57 & 0.3261 & 14.05 & 0.3538 & 25.29 & 0.3517 & 42.47 & 0.3577 & 79.42 & 0.3565 \\
\hline 5.57 & 0.3224 & 14.05 & 0.3465 & 25.29 & 0.3532 & 42.47 & 0.3559 & 79.42 & 0.3568 \\
\hline 5.57 & 0.3264 & 14.05 & 0.3471 & 25.29 & 0.3519 & 42.47 & 0.3555 & 79.42 & 0.3578 \\
\hline 5.57 & 0.3245 & 14.05 & 0.3505 & 25.29 & 0.3516 & 42.47 & 0.3553 & 79.42 & 0.3579 \\
\hline 5.57 & 0.3214 & 14.05 & 0.3479 & 25.29 & 0.3541 & 42.47 & 0.3586 & 79.42 & 0.3581 \\
\hline 5.57 & 0.3230 & 14.05 & 0.3495 & 25.29 & 0.3523 & 42.47 & 0.3578 & 79.42 & 0.3577 \\
\hline 5.57 & 0.3216 & 14.05 & 0.3510 & 25.29 & 0.3556 & 42.47 & 0.3569 & 79.42 & 0.3589 \\
\hline 5.57 & 0.3222 & 14.05 & 0.3529 & 25.29 & 0.3576 & 42.47 & 0.3567 & 79.42 & 0.3576 \\
\hline 5.57 & 0.3215 & 14.05 & 0.3484 & 25.29 & 0.3555 & 42.47 & 0.3580 & 79.42 & 0.3547 \\
\hline
\end{tabular}


Table S 7: Experimental data of stationary flow experiments with methanol and acetic acid.

\begin{tabular}{|c|c|c|c|c|c|c|c|c|c|}
\hline \multicolumn{2}{|c|}{$1.3 \mathrm{ma} \%$} & \multicolumn{2}{|c|}{$1.3 \mathrm{ma} \%$} & \multicolumn{2}{|c|}{$1.3 \mathrm{ma} \%$} & \multicolumn{2}{|c|}{$1.3 \mathrm{ma} \%$} & \multicolumn{2}{|c|}{$1.3 \mathrm{ma} \%$} \\
\hline $\mathrm{t} / \min$ & $\lambda$ & $\mathrm{t} / \min$ & $\lambda$ & $\mathrm{t} / \min$ & $\lambda$ & $\mathrm{t} / \min$ & $\lambda$ & $\mathrm{t} / \min$ & $\lambda$ \\
\hline 1.07 & 0.0247 & 4.85 & 0.0687 & 9.72 & 0.0992 & 16.55 & 0.1275 & 28.30 & 0.1658 \\
\hline 1.07 & 0.0257 & 4.85 & 0.0682 & 9.72 & 0.0973 & 16.55 & 0.1300 & 28.30 & 0.1638 \\
\hline 1.07 & 0.0259 & 4.85 & 0.0675 & 9.72 & 0.0946 & 16.55 & 0.1282 & 28.30 & 0.1662 \\
\hline 1.07 & 0.0262 & 4.85 & 0.0667 & 9.72 & 0.0981 & 16.55 & 0.1286 & 28.30 & 0.1680 \\
\hline 1.07 & 0.0270 & 4.85 & 0.0665 & 9.72 & 0.0970 & 16.55 & 0.1307 & 28.30 & 0.1667 \\
\hline 1.07 & 0.0257 & 4.85 & 0.0665 & 9.72 & 0.0985 & 16.55 & 0.1298 & 28.30 & 0.1680 \\
\hline 1.07 & 0.0272 & 4.85 & 0.0673 & 9.72 & 0.0977 & 16.55 & 0.1289 & 28.30 & 0.1668 \\
\hline 1.07 & 0.0255 & 4.85 & 0.0665 & 9.72 & 0.0959 & 16.55 & 0.1313 & 28.30 & 0.1644 \\
\hline 1.07 & 0.0267 & 4.85 & 0.0673 & 9.72 & 0.0957 & 16.55 & 0.1309 & 28.30 & 0.1665 \\
\hline 1.07 & 0.0269 & 4.85 & 0.0666 & 9.72 & 0.0946 & 16.55 & 0.1292 & 28.30 & 0.1669 \\
\hline 2.89 & 0.0454 & 7.14 & 0.0840 & 12.80 & 0.1096 & 21.44 & 0.1470 & 40.02 & 0.1952 \\
\hline 2.89 & 0.0482 & 7.14 & 0.0832 & 12.80 & 0.1098 & 21.44 & 0.1489 & 40.02 & 0.1925 \\
\hline 2.89 & 0.0453 & 7.14 & 0.0840 & 12.80 & 0.1104 & 21.44 & 0.1486 & 40.02 & 0.1924 \\
\hline 2.89 & 0.0477 & 7.14 & 0.0840 & 12.80 & 0.1109 & 21.44 & 0.1509 & 40.02 & 0.1919 \\
\hline 2.89 & 0.0462 & 7.14 & 0.0825 & 12.80 & 0.1134 & 21.44 & 0.1487 & 40.02 & 0.1927 \\
\hline 2.89 & 0.0445 & 7.14 & 0.0799 & 12.80 & 0.1099 & 21.44 & 0.1504 & 40.02 & 0.1941 \\
\hline 2.89 & 0.0479 & 7.14 & 0.0809 & 12.80 & 0.1094 & 21.44 & 0.1515 & 40.02 & 0.1927 \\
\hline 2.89 & 0.0471 & 7.14 & 0.0834 & 12.80 & 0.1124 & 21.44 & 0.1493 & 40.02 & 0.1930 \\
\hline 2.89 & 0.0467 & 7.14 & 0.0814 & 12.80 & 0.1122 & 21.44 & 0.1475 & 40.02 & 0.1950 \\
\hline 2.89 & 0.0465 & 7.14 & 0.0827 & 12.80 & 0.1108 & 21.44 & 0.1485 & 40.02 & 0.1972 \\
\hline \multicolumn{2}{|c|}{$3.5 \mathrm{ma} \%$} & \multicolumn{2}{|c|}{$3.5 \mathrm{ma} \%$} & \multicolumn{2}{|c|}{$3.5 \mathrm{ma} \%$} & \multicolumn{2}{|c|}{$3.5 \mathrm{ma} \%$} & \multicolumn{2}{|c|}{$3.5 \mathrm{ma} \%$} \\
\hline $\mathrm{t} / \min$ & $\lambda$ & $\mathrm{t} / \min$ & $\lambda$ & $\mathrm{t} / \min$ & $\lambda$ & $\mathrm{t} / \min$ & $\lambda$ & $\mathrm{t} / \min$ & $\lambda$ \\
\hline 1.07 & 0.0403 & 4.85 & 0.1358 & 9.72 & 0.1813 & 16.55 & 0.2253 & 28.30 & 0.2602 \\
\hline 1.07 & 0.0423 & 4.85 & 0.1358 & 9.72 & 0.1818 & 16.55 & 0.2283 & 28.30 & 0.2635 \\
\hline 1.07 & 0.0399 & 4.85 & 0.1371 & 9.72 & 0.1810 & 16.55 & 0.2290 & 28.30 & 0.2595 \\
\hline 1.07 & 0.0413 & 4.85 & 0.1335 & 9.72 & 0.1815 & 16.55 & 0.2244 & 28.30 & 0.2634 \\
\hline 1.07 & 0.0424 & 4.85 & 0.1339 & 9.72 & 0.1800 & 16.55 & 0.2264 & 28.30 & 0.2632 \\
\hline 1.07 & 0.0431 & 4.85 & 0.1374 & 9.72 & 0.1824 & 16.55 & 0.2277 & 28.30 & 0.2600 \\
\hline 1.07 & 0.0423 & 4.85 & 0.1379 & 9.72 & 0.1820 & 16.55 & 0.2284 & 28.30 & 0.2640 \\
\hline 1.07 & 0.0427 & 4.85 & 0.1385 & 9.72 & 0.1801 & 16.55 & 0.2234 & 28.30 & 0.2616 \\
\hline 1.07 & 0.0437 & 4.85 & 0.1378 & 9.72 & 0.1805 & 16.55 & 0.2270 & 28.30 & 0.2624 \\
\hline 1.07 & 0.0413 & 4.85 & 0.1384 & 9.72 & 0.1798 & 16.55 & 0.2258 & 28.30 & 0.2605 \\
\hline 2.89 & 0.1007 & 7.14 & 0.1637 & 12.80 & 0.2084 & 21.44 & 0.2406 & 40.02 & 0.2882 \\
\hline 2.89 & 0.1014 & 7.14 & 0.1660 & 12.80 & 0.2055 & 21.44 & 0.2402 & 40.02 & 0.2860 \\
\hline 2.89 & 0.1026 & 7.14 & 0.1604 & 12.80 & 0.2076 & 21.44 & 0.2403 & 40.02 & 0.2860 \\
\hline 2.89 & 0.1027 & 7.14 & 0.1651 & 12.80 & 0.2066 & 21.44 & 0.2426 & 40.02 & 0.2887 \\
\hline 2.89 & 0.1023 & 7.14 & 0.1613 & 12.80 & 0.2074 & 21.44 & 0.2396 & 40.02 & 0.2879 \\
\hline 2.89 & 0.1020 & 7.14 & 0.1635 & 12.80 & 0.2061 & 21.44 & 0.2402 & 40.02 & 0.2878 \\
\hline 2.89 & 0.1014 & 7.14 & 0.1609 & 12.80 & 0.2081 & 21.44 & 0.2446 & 40.02 & 0.2858 \\
\hline 2.89 & 0.1032 & 7.14 & 0.1617 & 12.80 & 0.2052 & 21.44 & 0.2449 & 40.02 & 0.2893 \\
\hline 2.89 & 0.1022 & 7.14 & 0.1599 & 12.80 & 0.2080 & 21.44 & 0.2417 & 40.02 & 0.2830 \\
\hline 2.89 & 0.1028 & 7.14 & 0.1586 & 12.80 & 0.2076 & 21.44 & 0.2423 & 40.02 & 0.2845 \\
\hline
\end{tabular}


Table S 8: Averaged mean values of experimental data of stationary flow experiments.

\begin{tabular}{|c|c|c|c|c|c|c|c|}
\hline \multicolumn{4}{|c|}{ Methanol + Formic acid } & \multicolumn{4}{|c|}{ Methanol + Acetic acid } \\
\hline \multicolumn{2}{|c|}{$0 \mathrm{ma} \% \mathrm{MW}$} & \multicolumn{2}{|c|}{$2 \mathrm{ma} \% \mathrm{MW}$} & \multicolumn{2}{|c|}{$1.3 \mathrm{ma} \% \mathrm{MW}$} & \multicolumn{2}{|c|}{$3.5 \mathrm{ma} \% \mathrm{MW}$} \\
\hline $\mathrm{t} / \min$ & $\lambda$ & $\mathrm{t} / \min$ & $\lambda$ & $\mathrm{t} / \min$ & $\lambda$ & $\mathrm{t} / \min$ & $\lambda$ \\
\hline 1.98 & 0.0436 & 1.98 & 0.2633 & 1.07 & 0.0261 & 1.07 & 0.0419 \\
\hline 5.57 & 0.0981 & 5.57 & 0.3235 & 2.89 & 0.0465 & 2.89 & 0.1021 \\
\hline 9.49 & 0.1355 & 9.49 & 0.3392 & 4.85 & 0.0672 & 4.85 & 0.1366 \\
\hline 14.05 & 0.1602 & 14.05 & 0.3500 & 7.14 & 0.0826 & 7.14 & 0.1621 \\
\hline 19.16 & 0.1800 & 19.16 & 0.3530 & 9.72 & 0.0969 & 9.72 & 0.1810 \\
\hline 25.29 & 0.1954 & 25.29 & 0.3535 & 12.80 & 0.1109 & 12.80 & 0.2070 \\
\hline 32.72 & 0.2123 & 32.72 & 0.3567 & 16.55 & 0.1295 & 16.55 & 0.2266 \\
\hline 42.47 & 0.2289 & 42.47 & 0.3568 & 21.44 & 0.1491 & 21.44 & 0.2417 \\
\hline 56.09 & 0.2393 & 56.09 & 0.3578 & 28.30 & 0.1663 & 28.30 & 0.2618 \\
\hline 79.42 & 0.2582 & 79.42 & 0.3573 & 40.02 & 0.1937 & 40.02 & 0.2867 \\
\hline
\end{tabular}

